\title{
Sugar-Sweetened Beverage Intake Assessment in a Family Medicine Residency Clinic
}

\author{
Jamil Neme, MD | Maya Nirmalraj, BS | Haley Matthews, BS | Jenenne Geske, PhD | Birgit \\ Khandalavala, MD
}

PRiMER. 2018;2:8.

Published: 3/26/2018 | DOI: 10.22454/PRiMER.2018.891960

\section{Abstract}

Introduction: Sugar-sweetened beverages (SSBs) are a major source of added calories in the American diet, with significant adverse health outcomes. However, intake of SSBs is not commonly assessed in the clinical setting. In some populations with health disparities there is a higher consumption of caloric beverages, contributing to increased cardiometabolic risk. Family medicine residency clinics often provide services for the underserved population, and may encounter additional barriers in nutritional assessment.

Methods: Our study, conducted at a residency clinic in Omaha, Nebraska, utilized the abbreviated Beverage Intake Questionnaire 15 (BEVQ-15). We surveyed 310 patients over a 3-month period.

Results: Consumption of SSBs in our sample was significantly higher than that of the nonclinic population of Nebraska $(P<0.001)$. Fifty-six percent of resident clinic respondents reportedly consumed at least one SSB daily, resulting in an average intake of 244 kilocalories. Intake was significantly higher in males, younger patients, those with lower socioeconomic status, and Latinos. While the majority of the study population was obese, there was no direct correlation between weight and SSB intake. The survey required less than 3 minutes to complete. Future interventions may focus on reduction of SSB intake and promotion of water consumption.

Conclusions: Assessment of SSB intake in a family medicine residency clinic can be easily conducted, providing opportunities for clinical providers to suggest patient-specific modifiable behaviors. As the obesity epidemic continues to progress, point-of-care intervention to reduce SSB consumption may help reduce cardiometabolic risk and improve health outcomes.

\section{Background}

Obesity is an ongoing pandemic of complex etiology. ${ }^{1}$ Sugar-sweetened beverages (SSBs) have been implicated since they are the primary source of added sugars in the American diet, ${ }^{2-4}$ contributing up to $50 \%$ of excess calories ${ }^{5}$ with lower satiety ${ }^{6}$ and adverse metabolic effects. ${ }^{2}$ Cardiovascular disease and type 2 diabetes may be increased independent of obesity. ${ }^{7}$ In addition, SSB consumption is reportedly higher in males, non-Hispanic blacks, and lowincome families, ${ }^{3}$ further aggravating undesirable health outcomes and costs. ${ }^{8}$

Reduction of consumption of SSBs has been found to be significantly beneficial, ${ }^{9}$ although direct causality has yet to be established. ${ }^{10}$ There is a gap in the assessment of SSB intake in the family medicine practice population using a formal validated tool. ${ }^{11}$ In addition, medical residents who practice in family medicine clinics may have additional barriers to optimal nutritional counseling, including patient health disparities and literacy limitations. ${ }^{12,13}$ The Beverage Intake Questionnaire 15 (BEVQ-15) is a brief measure of habitual SSB intake. ${ }^{14}$ It has been extensively 
validated in conjunction with the Food Frequency Questionnaire ${ }^{14,15,19}$ in our local population, ${ }^{15}$ nationally, and internationally. ${ }^{16-18}$

Our observational study was aimed at assessing the beverage consumption of adult patients in a family medicine residency clinic that has a predominantly underserved population, as the first step toward developing an intervention to mitigate consumption.

\section{Methods}

The BEVQ-15 quantifies the intake of SSBs, water, and alcohol, in fluid ounces. There are five categories of SSBs: regular soft drinks, tea/coffee with cream, sweetened juice drink, sweet tea, and energy drinks. Conversion into caloric energy was conducted using a key provided by the author of the survey. IRB clearance (375-5 EX) was obtained from the Institutional Review Board at the University of Nebraska Medical Center. Over a 3-month period, 310 patients were recruited by research assistants from the family medicine resident clinics. After patients were placed in an exam room and before the physician arrived, patients were approached by a research assistant. Patients who did not speak either English or Spanish were excluded. Following verbal consent, the patient received a copy of the survey. One patient declined the survey due to lack of interest.

\section{Results}

The study population was predominantly Caucasian and female, with a mean age of 47.9 years $(S D=16.5$, range 19-96 years, Table 1). A large majority (83.3\%) were overweight, obese or extremely obese, and insurance status was evenly distributed between private and others (Medicaid, Medicare or self-pay). On average, completion of the survey took less than 3 minutes. Regular soft drinks were the predominant caloric beverage consumed, followed by $100 \%$ juice and whole milk (Table 2). Average water consumption was 34.99 ounces per day.

Of the sample, $55.9 \%$ consumed one or more sugar-sweetened beverages daily. The average daily consumption of all beverages was 487.79 kilocalories ( $\mathrm{kcal} ; \mathrm{SD}=435.9$, range $0-2,516.5$ ) with $243.9 \mathrm{kcal}(\mathrm{SD}=278.2$, range $0-1,470$ ) from SSBs.

A higher intake of total beverages and SSBs was seen in males compared to females $(P<0.001$ and $P=0.002$, respectively, Table 1), and intake of total beverages and SSBs were found to decline with increasing age $(P=0.027$ and $P=0.049$, respectively). Overall beverage consumption was higher in the Latino subgroup when compared to whites $(P=0.040)$, but no ethnic differences were noted for SSB intake $(P=0.095)$. Insurance status was used as a surrogate for socioeconomic status; significantly higher caloric intake of all beverages $(P=0.010)$ and SSBs $(P=0.014)$ were noted in the group with Medicaid when compared to those with private insurance. There were no significant differences in total caloric intake of beverages from SSBs across BMI categories. Intake of total beverages $(r=-.126, P=0.027)$ and SSBs $(r=-.112, P=0.049)$ were found to decline with increasing age.

\section{Discussion}

To our knowledge, this is the first report of beverage intake using the BEVQ-15 in a resident teaching clinic population. The daily consumption of SSBs in our study sample (55.9\%) was significantly higher than the daily consumption of SSBs for the general population of Nebraska $(26.8 \% ; P<0.001) .{ }^{20}$ Our analysis revealed that younger adult men and those with assumed lower socioeconomic status had significantly higher consumption of SSBs than the other respondents in our study. Though a majority of our study group was overweight or obese, we did not detect a direct relationship with body weight. The American Heart Association recommends daily added-sugar intake less than 100-150 kcal. ${ }^{21}$ Hence, an average intake of 244 kcals from SSBs makes it a critical factor for mitigation. Substituting water for SSBs is encouraged. ${ }^{22}$

Strengths of our study include a large sample size and strong representation of minority groups. The BEVQ-15 is much shorter than most food frequency questionnaires; the completion time for our sample was similar to previous 
reports. ${ }^{15}$ The BEVQ-15 has a readability score of grade $4.8,{ }^{15}$ which is suited for a sample of patients who may have reduced literacy.

Study limitations include the typical constraints of self-reported data, including potential underreporting of SSB consumption due to social desirability bias and other factors. ${ }^{6}$ Since the majority of our study population was already overweight or obese, a direct relationship to BMI was difficult to examine. We did not include confounding factors such as smoking status or medications. Our results mirrored results of a Centers for Disease Control and Prevention study, which found the highest consumption rates of SSBs among younger men (18-24 years); however, this group is also the least likely to be seen in a family medicine clinic, ${ }^{20}$ which could affect the generalizability of our results. Future studies using a biomarker for SSB consumption, or using a computerized survey could provide more reliable results. ${ }^{23,24}$

The BEVQ-15 has been found to be valid and reliable. ${ }^{14,15}$ Its brevity allows clinic providers to rapidly assess SSB consumption which, in turn, enables them to provide targeted feedback specific to each patient. ${ }^{18,25,26}$ Attitudes and opinions of the providers about the usefulness of the tool's results could additionally be incorporated in prospective studies to enhance outcomes.

There is evidence that decreasing SSBs would help prevent obesity progression in children and adults, although short-term weight loss may not be evident. ${ }^{4,27}$ Based on our study, we recommend that residents be educated to include assessment of SSBs as part of a comprehensive clinical assessment, particularly in areas with large health disparities. Current resident physicians will be at the forefront in the treatment of the obesity epidemic and will require tools that are effective and efficient. Eventual translation into a timely intervention at the point of care to promote healthy drinking habits could contribute to improved cardiometabolic risk, and eventually to lower health care costs. $^{8}$

\section{Tables and Figures}


Table 1: Demographics and Average Kilocalories From Total Beverage Consumption and SSBs

\begin{tabular}{|c|c|c|c|c|}
\hline & Mean & SD & Range & \\
\hline \multirow[t]{2}{*}{ Age (Years) } & 47.86 & 16.5 & 19.96 & \\
\hline & $\mathbf{N}$ & $\begin{array}{l}\text { Percent of } \\
\text { Sample }\end{array}$ & $\begin{array}{c}\text { Total Kilocalories } \\
\text { Mean (SD) }\end{array}$ & $\begin{array}{c}\text { SSB Kilocalories } \\
\text { Mean (SD) }\end{array}$ \\
\hline Entire Sample & 310 & 100 & $487.8(435.9)$ & $243.9(278.2)$ \\
\hline \multicolumn{5}{|l|}{ Ethnicity/Race } \\
\hline Caucasian & 166 & 53.5 & $429.1(424.7)$ & $208.3(270.1)$ \\
\hline African American & 89 & 28.7 & $526.8(438.8)$ & $303.3(297.7)$ \\
\hline Hispanic or Latino & 25 & 8.1 & $696.6(520.4)$ & $268.0(254.9)$ \\
\hline Other & 11 & 3.5 & $463.1(353.4)$ & $180.5(258.6)$ \\
\hline More than one race identified & 19 & 6.1 & $557.6(363.4)$ & $281.8(262.5)$ \\
\hline \multicolumn{5}{|l|}{ Gender } \\
\hline Male & 116 & 37.4 & $633.5(479.0)$ & $306.2(318.0)$ \\
\hline Female & 194 & 62.6 & $400.7(383.6)$ & $206.6(244.7)$ \\
\hline \multicolumn{5}{|l|}{ Body Mass Index } \\
\hline Underweight & 3 & 1 & $390.9(280.2)$ & $91.3(158.2)$ \\
\hline Normal & 52 & 16.8 & $492.5(496.1)$ & $186.3(238.3)$ \\
\hline Overweight & 149 & 48.1 & 495.8 (395.9) & $253.8(267.5)$ \\
\hline Obese & 49 & 15.8 & $438.4(373.5)$ & $240.4(241.4)$ \\
\hline Extremely obese & 57 & 18.4 & $510.2(533.5)$ & $281.5(359.6)$ \\
\hline \multicolumn{5}{|l|}{ Insurance Status } \\
\hline Private & 155 & 50.2 & $434.3(396.8)$ & $205.5(270.0)$ \\
\hline Medicare & 37 & 12 & 401.9 (392.9) & 219.5 (261.9) \\
\hline Medicaid & 104 & 33.7 & $604.4(484.0)$ & $316.4(289.9)$ \\
\hline Self-pay & 13 & 4.2 & $447.7(468.2)$ & $206.8(242.6)$ \\
\hline
\end{tabular}


Table 2: Average Beverage Consumption in Fluid Ounces and Kilocalories per Day

\begin{tabular}{|c|c|c|}
\hline $\mathbf{N}=\mathbf{3 1 0}$ & Mean Fluid Ounces (SD) & Mean Kilocalories (SD) \\
\hline Water & $35.0(18.5)$ & $9.0(0.0)$ \\
\hline Regular soft drink $^{*}$ & $7.0(12.1)$ & $43.6(161.4)$ \\
\hline Tea/coffee with cream ${ }^{*}$ & $5.6(9.4)$ & $1.4(3.4)$ \\
\hline Diet soda & $4.7(11.2)$ & $1.5(3.4)$ \\
\hline Black coffee & $4.4(9.7)$ & $64.0(111.4)$ \\
\hline 100\% juice & $3.6(6.3)$ & $34.0(103.0)$ \\
\hline Sweet tea* & $3.4(10.3)$ & $30.5(88.3)$ \\
\hline Skim milk & $2.7(7.7)$ & $38.1(116.3)$ \\
\hline Sweetened juice drink & $2.7(8.1)$ & $57.5(170.4)$ \\
\hline Whole milk & $2.5(7.5)$ & $46.3(126.6)$ \\
\hline 2\% milk & $2.5(6.8)$ & $32.6(94.9)$ \\
\hline Energy drink & $2.3(6.8)$ & $16.5(39.3)$ \\
\hline Beer & $1.6(3.8)$ & $8.9(34.6)$ \\
\hline Wine & $0.4(1.7)$ & $17.3(61.8)$ \\
\hline Hard liquor & $0.3(0.9)$ & \\
\hline
\end{tabular}

*Sugar-sweetened beverages

\section{Acknowledgments}

We thank Dr Jacob Prunuske for his assistance and comments that greatly improved the manuscript.

\section{Corresponding Author}

Birgit Khandalavala, MD

University of Nebraska Medical Center, Department of Family Medicine, 983075 Nebraska Medical Center, Omaha, NE 68198-3075. 402-559-3864. Fax: 402-559-6051

birgit.khandalavala@unmc.edu

\section{Author Affiliations}

Jamil Neme, MD - Department of Primary Care Sports Medicine, Saint Vincent Hospital, Allegheny Health Network, Erie, PA

Maya Nirmalraj, BS - Department of Family Medicine, University of Nebraska Medical Center, Omaha, NE Haley Matthews, BS - Department of Family Medicine, University of Nebraska Medical Center, Omaha, NE Jenenne Geske, PhD - Department of Family Medicine, University of Nebraska Medical Center, Omaha, NE Birgit Khandalavala, MD - Department of Family Medicine, University of Nebraska Medical Center, Omaha, NE

\section{References}

1. Flegal KM, Kruszon-Moran D, Carroll MD, Fryar CD, Ogden CL. Trends in obesity among adults in the united states, 2005 to 2014. JAMA. 2016;315(21):2284-2291. https://doi.org/10.1001/jama.2016.6458.

2. Malik VS, Popkin BM, Bray GA, Després JP, Hu FB. Sugar-sweetened beverages, obesity, type 2 diabetes mellitus, and cardiovascular disease risk. Circulation. 2010;121(11):1356-1364. https://doi.org/10.1161 /CIRCULATIONAHA.109.876185.

3. Centers for Disease Control and Prevention. Get the facts: Sugar-sweetened beverages and consumption. https://www.cdc.gov/nutrition/data-statistics/sugar-sweetened-beverages-intake.html. Updated 2017. Accessed March 14, 2018.

4. Hu FB. Resolved: there is sufficient scientific evidence that decreasing sugar-sweetened beverage 
consumption will reduce the prevalence of obesity and obesity-related diseases. Obes Rev. 2013;14(8):606-619. https://doi.org/10.1111/obr.12040.

5. Briefel RR, Johnson CL. Secular trends in dietary intake in the United States. Annu Rev Nutr. 2004;24(1):401-431. https://doi.org/10.1146/annurev.nutr.23.011702.073349.

6. Pan A, Hu FB. Effects of carbohydrates on satiety: differences between liquid and solid food. Curr Opin Clin Nutr Metab Care. 2011;14(4):385-390. https://doi.org/10.1097/MC0.0b013e328346df36.

7. Fung TT, Malik V, Rexrode KM, Manson JE, Willett WC, Hu FB. Sweetened beverage consumption and risk of coronary heart disease in women. Am J Clin Nutr. 2009;89(4):1037-1042. https://doi.org/10.3945 /ajcn.2008.27140.

8. Cawley J, Meyerhoefer C. The medical care costs of obesity: an instrumental variables approach. J Health Econ. 2012;31(1):219-230. https://doi.org/10.1016/j.jhealeco.2011.10.003.

9. O'Connor L, Imamura F, Lentjes MA, Khaw KT, Wareham NJ, Forouhi NG. Prospective associations and population impact of sweet beverage intake and type 2 diabetes, and effects of substitutions with alternative beverages. Diabetologia. 2015;58(7):1474-1483. https://doi.org/10.1007/s00125-015-3572-1.

10. Forshee RA, Anderson PA, Storey ML. Sugar-sweetened beverages and body mass index in children and adolescents: a meta-analysis. Am J Clin Nutr. 2008;87(6):1662-1671. https://doi.org/10.1093/ajcn/87.6.1662.

11. Weed DL, Althuis MD, Mink PJ. Quality of reviews on sugar-sweetened beverages and health outcomes: a systematic review. Am J Clin Nutr. 2011;94(5):1340-1347. https://doi.org/10.3945/ajcn.111.015875.

12. Eaton CB, Goodwin MA, Stange KC. Direct observation of nutrition counseling in community family practice. Am J Prev Med. 2002;23(3):174-179. https://doi.org/10.1016/S0749-3797(02)00494-4.

13. Rodgers DV, Wendling AL, Saba GW, Mahoney MR, Brown Speights JS. Preparing family physicians to care for underserved populations. Fam Med. 2017;49(4):304-310.

14. Hedrick VE, Comber DL, Estabrooks PA, Savla J, Davy BM. The beverage intake questionnaire: determining initial validity and reliability. J Am Diet Assoc. 2010;110(8):1227-1232. https://doi.org/10.1016 /j.jada.2010.05.005.

15. Hedrick VE, Savla J, Comber DL, et al. Development of a brief questionnaire to assess habitual beverage intake (BEVQ-15): sugar-sweetened beverages and total beverage energy intake. J Acad Nutr Diet. 2012;112(6):840-849. https://doi.org/10.1016/j.jand.2012.01.023.

16. Karabudak E, Koksal E. Validity and reliability of beverage intake questionnaire: evaluating hydration status. Nutr Hosp. 2016;33(5):577.

17. Hedrick VE, Davy BM, Myers EA, You W, Zoellner JM. Changes in the healthy beverage index in response to an intervention targeting a reduction in sugar-sweetened beverage consumption as compared to an intervention targeting improvements in physical activity: results from the talking health trial. Nutrients.

2015;7(12):10168-10178. https://doi.org/10.3390/nu7125525.

18. Myers E, Fausnacht A, Brooks A, Hess E, Bremer M, Hedrick V. The ability of a beverage intake questionnaire (BEVQ-15) to rapidly assess healthy beverage index (HBI) scores among a diverse adult population. J Acad Nutr Diet. 2017;117(9)(suppl):A33. https://doi.org/10.1016/j.jand.2017.06.283.

19. Elwan D, Schweinitz P, Wojcicki JM. Beverage consumption in an Alaska Native village: a mixed-methods study of behaviour, attitudes and access. Int J Circumpolar Health. 2016;75(1):29905. https://doi.org/10.3402 /ijch.v75.29905.

20. Park S, Xu F, Town M, Blanck HM. Prevalence of sugar sweetened beverage intake among adults - 23 states and the district of columbia, 2013. MMWR Morb Mortal Wkly Rep. 2016;65(7):169-174. https://doi.org /10.15585/mmwr.mm6507a1.

21. Johnson RK, Appel LJ, Brands M, et al; American Heart Association Nutrition Committee of the Council on Nutrition, Physical Activity, and Metabolism and the Council on Epidemiology and Prevention. Dietary sugars intake and cardiovascular health: a scientific statement from the American Heart Association. Circulation. 2009;120(11):1011-1020. https://doi.org/10.1161/CIRCULATIONAHA.109.192627.

22. Centers for Disease Control. Get the facts: Drinking water and intake. https://www.cdc.gov/nutrition/datastatistics/plain-water-the-healthier-choice.html. Updated 2017. Accessed October 25, 2017.

23. Davy BM, Jahren AH, Hedrick VE, Comber DL. Association of $\delta^{13} \mathrm{C}$ in fingerstick blood with added-sugar and sugar-sweetened beverage intake. J Am Diet Assoc. 2011;111(6):874-878. https://doi.org/10.1016 
/j.jada.2011.03.019.

24. Nayak MB, Korcha RA, Kaskutas LA, Avalos LA. Feasibility and acceptability of a novel, computerized screening and brief intervention (SBI) for alcohol and sweetened beverage use in pregnancy. BMC Pregnancy Childbirth. 2014;14(1):379. https://doi.org/10.1186/s12884-014-0379-x.

25. Hill CE, MacDougall CR, RiebI SK, Savla J, Hedrick VE, Davy BM. Evaluation of the relative validity and TestRetest reliability of a 15 -item beverage intake questionnaire in children and adolescents. J Acad Nutr Diet. 2017;117(11):1757-1766.e5. https://doi.org/10.1016/j.jand.2017.05.011.

26. Dooley D, Moultrie NM, Sites E, Crawford PB. Primary care interventions to reduce childhood obesity and sugar-sweetened beverage consumption: food for thought for oral health professionals. J Public Health Dent. 2017;77(S1)(suppl 1):S104-S127. https://doi.org/10.1111/jphd.12229.

27. Vargas-Garcia EJ, El Evans C, Cade JE. Impact of interventions to reduce sugar-sweetened beverage intake in children and adults: a protocol for a systematic review and meta-analysis. Syst Rev. 2015;4(1):17.

https://doi.org/10.1186/s13643-015-0008-4.

Copyright $(\subset) 2018$ by the Society of Teachers of Family Medicine 